\title{
Myositis - die Vielfalt wird neu verstanden
}

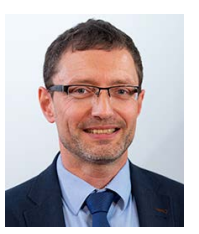

Eugen Feist
Bibliography

Akt Rheumatol 2021; 46: 305

DOI 10.1055/a-1468-9296

ISSN $0341-051 X$

(c) 2021. Thieme. All rights reserved.

Georg Thieme Verlag, Rüdigerstraße 14, 70469 Stuttgart, Germany

\author{
Korrespondenzadresse \\ Prof. Dr. med. Eugen Feist \\ Klinik für Rheumatologie \\ Helios Fachklinik Vogelsang-Gommern GmbH \\ Sophie-von-Boetticher-Straße 1 \\ 39245 Vogelsang \\ Eugen.Feist@helios-gesundheit.de
}

Seit unserem letzten Themenheft zu den „Idiopathischen inflammatorischen Myopathien “, die wir Rheumatologen auch gerne als „Myositiden“ bezeichnen, haben sich bei diesem interdisziplinären Krankheitsspektrum sehr viele neue Erkenntnisse durchgesetzt. Darauf begründet, habe ich den vormaligen Titel meines Editorials „...die Vielfalt wird neu geordnet“ nunmehr in „...die Vielfalt wird neu verstanden “ abgeändert.

In dieser Ausgabe der Aktuellen Rheumatologie werden nicht nur aktuelle und relevante Informationen für den Kliniker zum Thema der Myositiden zusammengefasst, sondern auch spannende Ausblicke in zukünftige Entwicklungen geboten. Im Beitrag von Udo Schneider, Werner Stenzel und Bruno Stuhlmüller dürfen wir die Thematik der Biomarker und Histologie bei idiopathischen inflammatorischen Myopathien gleichzeitig aus den Blickwinkeln eines Klinikers, Pathologen und Biologen betrachten. Diese Zusammensetzung hat das Verfassen des Beitrages, wie ich erfahren durfte, nicht leichter aber für uns als Leser umso wertvoller gemacht. Denn es macht für uns ein frappierend klares System der Zuordnung und Wechselwirkung zwischen Immunpathogenese, Immunbiomarkern und Immunhistologie erkennbar.

Darauf aufbauend schließt sich der Beitrag aus meiner Klinik mit Autorenschaft der Herrn Stylianos Tomaras, Jörn Kekow und Eugen Feist zum Thema Idiopathische inflammatorische Myopathien: Aktuelles zu Diagnose und Klassifikation an. Dabei wird ausgehend von einer genauen klinischen Untersuchung ein praxisnahes Vorgehen aufgezeigt. Weiterhin werden aber auch die aktuellen Klassifikationskriterien im Detail vorgestellt, ohne die bestehenden Limitationen auszublenden.
Da in diesem Kontext auch immer eine Abgrenzung zu anderen Fachgebieten wichtig ist, freut es mich sehr, dass wir mit Maren Fitzner und Jens Schmidt und mit Linda Golle und Cord Sunderkoetter über unseren Tellerrand als Rheumatologen schauen dürfen. In Ihrem exzellenten Beitrag zu Ursache von Muskelschwäche: Spektrum neuromuskulärer Erkrankungen und diagnostisches Vorgehen und Dermatologische Differentialdiagnosen von idiopathischen entzündlichen Muskelerkrankungen helfen Sie uns das Symptom der Muskelschwäche umfänglicher zu verstehen sowie Hautveränderungen besser einzuordnen und somit unsere Differentialdiagnostik abzurunden.

Im Beitrag von Leonore Unger werden uns abschließend die neuesten Informationen zu Therapieoptionen und outcome bei idiopathischen entzündlichen Muskelerkrankungen in prägnanter Form vorgestellt. Lesen Sie selbst- hier finden sich erfreulicherweise sehr viele neue Entwicklungen. Überhaupt ist frischer Wind und man kann sogar sagen eine Aufbruchsstimmung in der Myositis-Community zu spüren. Für mich ein sicheres Anzeichen dafür ist die Formierung von nunmehr seit Jahren zunehmend aktiven, interdisziplinären Kooperationen, wie z. B. in Deutschland in Form des MYOSITIS NETZ (Myositis - Wir forschen gemeinsam (myositis-netz.de)). Denn neben den inzwischen etablierten Kriterien und Messinstrumenten sowie einer Vielzahl von vielversprechenden Therapieansätzen ist die Organisation der Community mit Bildung von und Austausch zwischen spezialisierten Zentren eine Grundvoraussetzung für eine erfolgreiche Weiterentwicklung, um nicht zu sagen für einen anzustrebenden Durchbruch, in der Versorgung der idiopathischen inflammatorischen Myopathien. 